\title{
THE MINIMUM MASS FOR A DWARF GALAXY
}

\author{
J. Bland-Hawthorn ${ }^{1}$, R. Sutherland ${ }^{2}$ and T. Karlsson ${ }^{3}$
}

\begin{abstract}
A widely held view is that a "mini halo" with much less than $10^{8} M_{\odot}$ in dark matter is unlikely to retain any baryons because even a single SN event is expected to sweep out all of the gas. But we show that a clumpy medium is much less susceptible to SN sweeping (particularly if it is off-centred) because the coupling efficiency of the explosive energy is much lower than for a diffuse interstellar medium. With the aid of the sophisticated 3D hydro code Fyris, we show that baryons are retained and stars are formed in dark matter haloes down to $3 \times 10^{6} M_{\odot}$. The gas survives the SN explosion and is enriched with specific abundance yields of the discrete events. The smallest galaxies may not contribute a large fraction of baryons and dark matter to the formation of galaxies. But they are likely to carry important chemical signatures that were laid down in the earliest epochs of star formation, as we show. We discuss the results in light of the newly discovered, very metal poor, damped $\operatorname{Ly} \alpha$ systems and ultra-faint dwarf galaxies.
\end{abstract}

\section{Introduction}

One of the most important problems in astrophysics today is what constituted the earliest baryonic objects, and whether relics of these systems survive to the present day. So what were the first stellar objects? Was this exclusively the domain of the most massive stars, or did these occur within clusters comprising even greater numbers of intermediate or low mass stars (Tsuribe \& Omukai 2008; Clark et al. 2008), and do any of these stars survive to the present day (Okrochkov \& Tumlinson 2010)? There are few if any reliable observational constraints thus far. Here we introduce a new class of models to demonstrate that the minimum mass

\footnotetext{
${ }^{1}$ Sydney Institute for Astronomy, University of Sydney, School of Physics A28, NSW 2006, Australia

${ }^{2}$ Research School of Astronomy \& Astrophysics, Australian National University, Cotter Rd., Weston, ACT 2611, Australia

3 Sydney Institute for Astronomy, University of Sydney, School of Physics A28, NSW 2006, Australia
} 
of a dwarf galaxy that formed in the early universe may be considerably less than has been assumed to date.

Prior to the reionization epoch, it is generally believed that the first structures were low mass objects, $M_{J} \sim 10^{5} M_{\odot}$, where $M_{J}$ is the cosmological Jeans mass which is roughly independent of redshift prior to the reionization epoch (Peebles 1968). Primordial "minimum mass" objects are important to identify at any redshift because these may retain chemical signatures of the first and second generations of stars (Karlsson et al. 2011). Numerical simulations show that the earliest baryonic systems may have contained dark matter; this allowed the gas to cool in order to form the first star (e.g. Abel et al. 2002) ${ }^{1}$. After reionization, it is often stated that the minimum halo mass to retain any baryons must have exceeded $10^{8} M_{\odot}$ (e.g. Efstathiou 1992). These arguments based on ionization balance and heating find support from limited 3D numerical simulations (Gnedin 2000; Okamoto et al. 2008).

We stress that these earlier simulations do not completely exclude ongoing star formation during and after the reionization epoch. The calculations do not consider the time it takes for the gas to evaporate from the low mass halos. For a cosmic ionizing UV intensity $J_{0}=10^{-21} \mathrm{erg} \mathrm{cm} \mathrm{cm}^{-2} \mathrm{~s}^{-1} \mathrm{~Hz}^{-1} \mathrm{sr}^{-1}$, with a moderate power-law form of $f_{\nu} \propto \nu^{-2}$, we obtain an ionizing flux of $\sim 2 \times 10^{5}$ photons $\mathrm{cm}^{-2} \mathrm{~s}^{-1}$. The potential rate of evaporation of hydrogen atoms over the surface of a confined gas cloud, of radius $50 \mathrm{pc}\left(\sim 3 \times 10^{41} \mathrm{~cm}^{2}\right)$, assuming one ionizing photon evaporates one hydrogen atom, is then: $\dot{m}_{\text {evap }} \sim 1.5 \times 10^{-3}\left(n_{\mathrm{r}}+1\right)^{-1} M_{\odot} \mathrm{yr}^{-1}$, where the $n_{\mathrm{r}}$ term allows for the number of recombinations in the escaping wind. Thus, the timescale for evaporating $\sim 10^{4-5} M_{\odot}$ of gas in the models investigated below $\left(\tau_{\text {evap }} \sim f_{b} M_{\text {min }} / \dot{m}_{\text {evap }}\right)$ can exceed $10^{8}$ yr, which is long enough for star formation to proceed in the interior while the outer regions are evaporating. Here $f_{b}$ is the baryon fraction by mass within the dwarf galaxy with virial mass $M_{\min }$.

\section{Fyris simulations}

\subsection{New models}

Here we investigate the conditions under which a "minimum mass" stellar system confined by a dark-matter halo survives to the present day. We consider feedback arising from high-mass stars due to their stellar winds, their radiation fields, and their subsequent supernova explosions. Previous mechanical feedback models by MacLow \& Ferrara (1999) give a similar mass limit $\left(\gtrsim 10^{8} M_{\odot}\right)$ but were these $2 \mathrm{D}$ axisymmetric models with central explosions and smooth media. Their models overestimate the coupling of the SN energy and the surrounding medium.

\footnotetext{
${ }^{1}$ Peebles \& Dicke (1968) considered the possibility that the first structures were fully baryonic, more specifically, globular clusters that formed before the first galaxies came together. How globular clusters fit into the early history of galaxy formation remains a mystery. We do not consider them further because their enhanced metallicities $[\mathrm{Fe} / \mathrm{H}]>-2$ may obscure the signatures of early star formation (although see Pancino et al. 2010).
} 
We find that these limiting cosmological systems conceivably had total masses of $M_{\min } \sim 3 \times 10^{6} M_{\odot}$, well below the limit of what is thought to have survived the reionization epoch. We perform new calculations of chemical evolution within these "minimum mass" galaxies over cosmic time. In principle, we can hope to learn about the star formation before, during and immediately after the reionization epoch. Our models may even explain some of the heterogeneous group of objects labelled "ultra-faint dwarfs" (Martin et al. 2008), and new results from very metal poor DLAs (Cooke et al. 2010), which we discuss.

In our new models, the dark matter is described by a spherical Einasto function with three different masses: $1 \times 10^{6} M_{\odot}(\mathrm{M} 60), 3 \times 10^{6} M_{\odot}(\mathrm{M} 65), 1 \times 10^{7} M_{\odot}$ (M 70). The baryon gas fraction is of order $f_{b} \approx 3-5 \%$ which is much less than the cosmological fraction (averaged on Mpc scales) of $17 \%$. This is consistent with observations of all dark-matter dominated systems observed today (McGaugh et al. 2010) and is expected on theoretical grounds (Barkana \& Loeb 1999; Gnedin 2000). The Fyris ionization hydrodynamics code is used throughout (Bland-Hawthorn et al. 2007; Sutherland 2010). For each model, we consider four treatments of the gas phase: adiabatic smooth medium, cooling smooth medium, adiabatic fractal medium, cooling fractal medium. We also consider a central and an off-centre SN explosion. The detailed results of all simulation are presented in Bland-Hawthorn et al. (2011); here we give only a brief overview of some key results.

\subsection{Gas retention}

A sample of our results is presented in Figure 1 and Table 1. Our main findings are:

1. no M 60 model retains significant gas mass within a scale radius $\left(r_{s}\right)$;

2. none of the smooth adiabatic models retain significant gas, consistent with earlier work;

3. cooling is very important in smooth models, but less so in clumpy models, for gas retention;

4. clumpiness and/or off-centred explosions lead to the highest gas retention fractions:

5. M 65 provides a conservative lower mass limit $\left(M_{\min } \gtrsim 3 \times 10^{6} M_{\odot}\right)$ for a halo that retains significant baryons.

For all models, the cold baryonic matter is centrally concentrated, and while it takes up about $10 \%$ by mass in the inner scale radius, it comprises approximately $1 \%$ out to the virial radius. The M 70 model has a gas distribution that is very centrally condensed compared to the dark matter halo (e.g. Fig. 1(v)); in contrast, we find the M 65 models to be more diffuse.

In Table 1, we show the fraction of gas retained in both central and off-centred explosions. Off-centred explosions are to be expected over an extended 

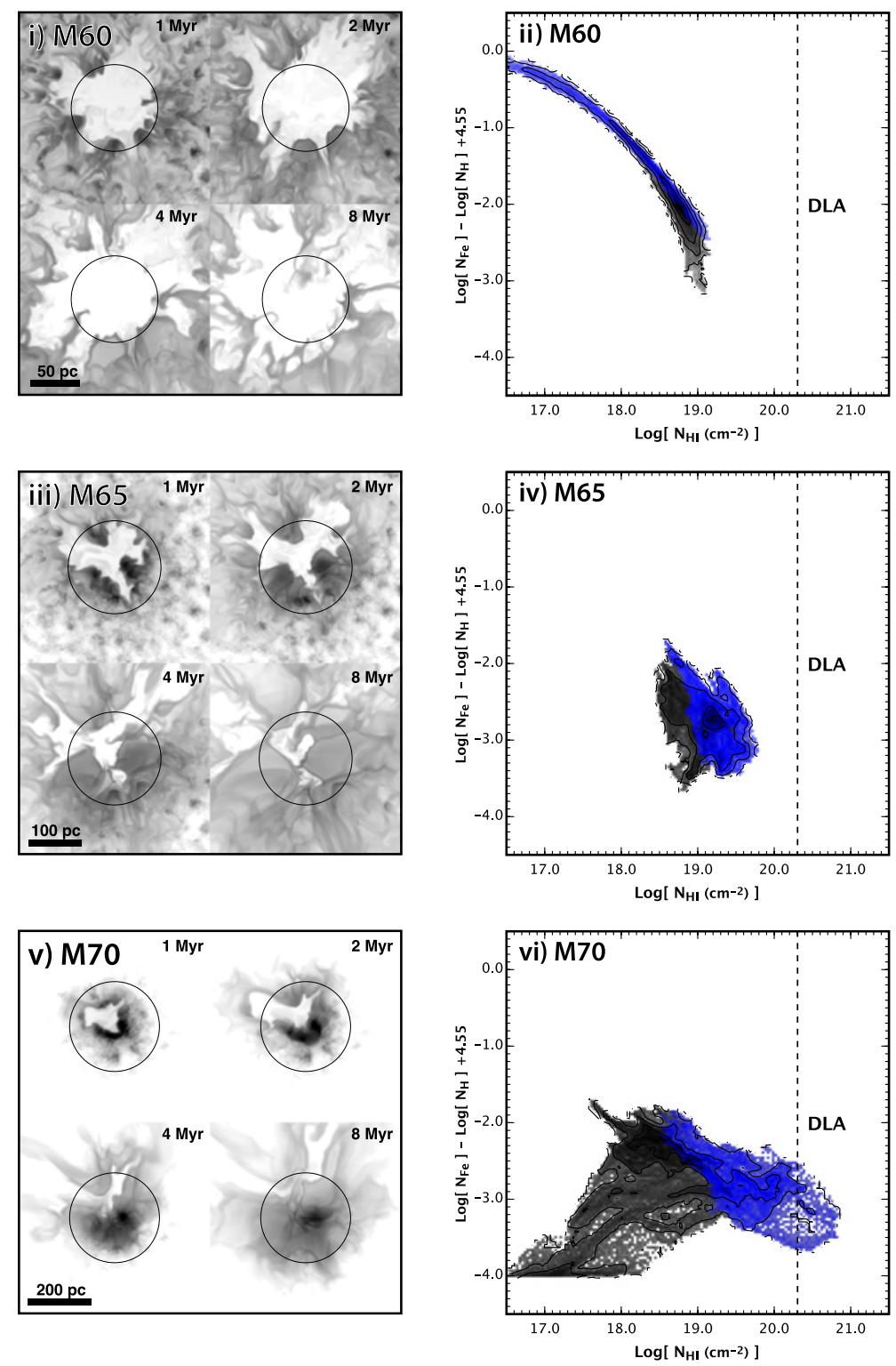

Fig. 1. Projected gas density for central explosions in a cooling clumpy medium at 4 different times: (i) $\mathrm{M} 60$; (ii) $\mathrm{N}_{\mathrm{HI}}$ vs. $[\mathrm{Fe} / \mathrm{H}]$ from (i); (iii) $\mathrm{M} 65$; (iv) $\mathrm{N}_{\mathrm{HI}}$ vs. $[\mathrm{Fe} / \mathrm{H}]$ from (iii); (v) M 70; (vi) $\mathrm{N}_{\mathrm{HI}}$ vs. [Fe/H] from (v). In panels (i), (iii), (v), the circle denotes the scale radius $r_{s}$ which scales with the mass of the dark matter halo. In panels (ii), (iv), (vi), the halftone region is averaged over $r<2 r_{s}$ and the blue region is averaged over $r<r_{s}$; the vertical dashed line indicates the minimum column density of a DLA system. 
Table 1. Gas fractions retained at $t=10$ Myr for the three mass models M 60, M 65 and M 70 within different radial bounds. Values exceeding $10 \%$ are shown in bold. The first two columns are for central SN explosions in a clumpy medium; the next four columns are for off-centred explosions for both smooth and clumpy media.

\begin{tabular}{|c|c|c|c|c|c|c|c|}
\hline \multirow{3}{*}{$\begin{array}{l}\text { Scale } \\
\text { Radii }\end{array}$} & \multirow{3}{*}{$\begin{array}{l}\text { Mass } \\
\text { Model }\end{array}$} & \multirow{2}{*}{\multicolumn{2}{|c|}{$\begin{array}{l}\text { Central } \\
\text { Clumpy }\end{array}$}} & \multicolumn{4}{|c|}{ Off-Centre } \\
\hline & & & & \multicolumn{2}{|c|}{ Smooth } & \multicolumn{2}{|c|}{ Clumpy } \\
\hline & & Adiabat & Cooling & Adiabat & Cooling & Adiabat & Cooling \\
\hline \multirow[t]{3}{*}{$1.0 r_{s}$} & M 60 & $2.2 \mathrm{e}-3$ & $4.1 \mathrm{e}-3$ & $1.0 \mathrm{e}-2$ & $3.9 \mathrm{e}-3$ & $3.1 \mathrm{e}-2$ & $3.6 \mathrm{e}-2$ \\
\hline & M 65 & $2.3 \mathrm{e}-2$ & $9.4 \mathrm{e}-2$ & $1.4 \mathrm{e}-1$ & $3.8 \mathrm{e}-1$ & $2.4 \mathrm{e}-1$ & $4.4 \mathrm{e}-1$ \\
\hline & M 70 & $3.1 \mathrm{e}-1$ & $6.7 \mathrm{e}-1$ & $8.2 \mathrm{e}-1$ & $9.1 \mathrm{e}-1$ & 8.3e-1 & $9.1 \mathrm{e}-1$ \\
\hline \multirow[t]{3}{*}{$2.0 r_{s}$} & M 60 & $2.4 \mathrm{e}-2$ & $3.4 \mathrm{e}-2$ & $3.5 \mathrm{e}-2$ & $6.0 \mathrm{e}-2$ & $8.2 \mathrm{e}-2$ & $1.3 \mathrm{e}-1$ \\
\hline & M 65 & $1.4 \mathrm{e}-1$ & $3.4 \mathrm{e}-1$ & $3.5 e-1$ & $6.3 e-1$ & $4.2 \mathrm{e}-1$ & $6.0 \mathrm{e}-1$ \\
\hline & M 70 & $6.2 \mathrm{e}-1$ & $9.1 \mathrm{e}-1$ & $9.1 \mathrm{e}-1$ & $9.6 \mathrm{e}-1$ & $9.2 \mathrm{e}-1$ & $9.6 e-1$ \\
\hline
\end{tabular}

star-forming region. The asymmetric gas distribution then leads to much of the supernova energy escaping through half the available solid angle. It is striking how most of the gas is retained in the M 70 models, with about half the gas in the M 65 models, within 2 scale radii. In the off-centred explosions, where the SN is placed at $1 r_{s}$, there is significant gas retention even when the gas is smoothly distributed.

The densities in the outer halo, even of warm gas, are very low and the cooling timescales (and hence time to start reforming cold gas in the centre) become much longer than the evolutionary timescales considered here ( $\sim 10 \mathrm{Myr})$. Our models consider dwarf galaxies to be isolated, but in practice the vast majority reside in the extended halo of a host galaxy. We explore environmental issues in later work but these are more important for more massive dwarf systems (Nichols \& Bland-Hawthorn 2009). We address the possibility that expelled gas can be re-accreted at a later time. This requires that we run our simulations for an order of magnitude longer.

\subsection{Mixing of metals}

The clumpy off-centred supernova models retain more gas mass for a given halo mass. But for these, most of the metals escape rather than mix with the dense gas in the nuclear regions. This is in contrast to the centred supernova models where the metals mix much more easily with the surrounding dense gas. However, the core regions are now more easily evacuated, and the hydrogen column densities are reduced. With a view to recent discoveries in very metal poor, damped Ly $\alpha$ (DLA) systems (Sect. 3), we now look at the metal distribution in more detail. 
In Figure 1, we present the projected distribution of hydrogen column density vs. $[\mathrm{Fe} / \mathrm{H}]$ for the clumpy models. The vertical dashed line indicates the minimum column density that defines the class of DLA systems. After $10 \mathrm{Myr}$, there are no sight lines in the M 60 and M 65 models that would reach the DLA threshold. But the M 70 models fair better and, in fact, most sight lines within $r<r_{s}$ would be classed as a DLA-type spectrum. This gas is mostly self-shielded from any external radiation and therefore does not require an ionization correction.

The M 60 models show a tight correlation between gas column density and metallicity because all that is seen here is the doomed gas that has yet to escape from the dark-matter halo. The central explosion model extends to high metallicity and low gas density which shows that most of the metals are escaping in a hot wind. The off-centre explosion model extends down to low metallicity and high gas density: this gas is on the far side from the explosion and is relatively unpolluted even while doomed to escape the halo.

The M 65 models show some evidence of metals escaping in the off-centre explosion, but also that the far-side gas remains relatively unpolluted (and mostly retained). The central explosion here constitutes an interesting case. The gas is highly concentrated in $\mathrm{HI}$ column density and $[\mathrm{Fe} / \mathrm{H}]$ which shows that the retained gas has soaked up most of the energy and metals. In consequence, the gas is well mixed but falls below the DLA limit.

The M 70 models feature prominently in our discussion of very metal poor DLAs in the next section. The off-centre model reveals enriched low-density gas due to leaking hot gas, and untouched gas at low metallicity. This is to be compared to the central explosion model where the gas close to the metallicity floor has now been raised to $[\mathrm{Fe} / \mathrm{H}] \sim-3$. Note also in Figure $1(\mathrm{vi})$ that there are sight lines enriched to this level that would have been "selected" as DLA objects.

\section{Discussion}

Minimum mass (or threshold) galaxies lie at the bottom of the CDM hierarchy. But our focus here is not their role as building blocks in galaxy evolution, but what they can tell us about the yields of the first stars prior to the reionization epoch, and the following generations during and immediately after reionization. If we can identify such objects, it should be possible to obtain a relatively clean signature of the primordial yields from the earliest stellar generations. The star-formation efficiency is greatly diminished in low-mass galaxies (Tolstoy et al. 2009), thereby reducing the total number of enrichment events. There are likely to be instances where a large fraction of the metals are blown out of the galaxy, and this may obscure the signatures of earlier generations of stars. This can occur when the relatively rare supernovae that go off in these galaxies are correlated in time. But we would still expect to find the signatures of a single enrichment event in at least some intrinsically low-mass galaxies.

We anticipate two observational approaches to identifying minimum mass galaxies or their consequences. The first is from the surviving star clusters in the local universe, in particular, ultra-faint dwarf galaxies (UFDs) recently identified by the 
Sloan survey. The second is from very metal poor DLA systems observed along QSO sight lines. We briefly discuss each of these in turn.

In recent years, the Sloan Digital Sky Survey has greatly increased the number of known dwarf galaxies that orbit the Galaxy (for a review, see Tolstoy et al. 2009). These newly discovered dark-matter dominated galaxies are uniformly very old and cover a wide range in luminosity down to $\sim 10^{3} L_{\odot}$. Unlike globular clusters, they show a wide range of eccentricities which may indicate that some are undergoing tidal disruption (Martin et al. 2008). There has been extensive discussion about their likely virial masses, with most studies concluding that the mass within $300 \mathrm{pc}$ is $\mathrm{M}_{300} \approx 10^{7} M_{\odot}$ (Strigari et al. 2008; Walker et al. 2009). Interestingly, this may indicate that for UFDs, $\mathrm{M}_{\mathrm{vir}} \approx 3 \mathrm{M}_{300}$ or about $3 \times 10^{7} M_{\odot}$, a mass that exceeds our most massive halo. Thus, if UFD masses are largely unchanged since their early formation, we would expect these halos to show significant scatter in $[\mathrm{Fe} / \mathrm{H}]$ at low metallicity, and for some of the oldest stars to constrain the yields of early stellar generations.

An interesting question is whether the low dark-matter halo masses inferred for the ultra-faint dwarf galaxies reflect the masses at the time of their formation, or whether these have been stripped down from larger systems. The abundance scatter that we observe in Figure 1(vi) is consistent with the scatter now observed in some ultra-faint dwarf galaxies (e.g. Kirby et al. 2010). With a sufficiently complete model, it may be possible to distinguish between the two cases above.

Another interesting development is the recent discovery of very metal-poor DLAs along QSO sight lines (Pettini et al. 2008; Penprase et al. 2010). If these are protogalactic structures that have recently formed from the IGM, there is the prospect of identifying the chemical imprint of early generations of stars (e.g. Pettini et al. 2002). Interestingly, two of these systems with $[\mathrm{Fe} / \mathrm{H}] \sim-3$ may bear the hallmarks of early stellar enrichment (Erni et al. 2006; Cooke et al. 2010).

First, Erni et al. (2006) identify a DLA toward the QSO Q0913+072 $(z=$ $2.785)$ with an iron abundance characteristic of the IGM at that redshift. The $\mathrm{C}, \mathrm{N}, \mathrm{O}, \mathrm{Al}, \mathrm{Si}$ abundances show an odd-even effect reminiscent of the most metal-poor stars in the Galactic halo. This pattern is created in models where the neutron flux is low (e.g. Heger \& Woosley 2002), presumably due to the low overall metal abundance. A more striking signature is the strong $[\mathrm{N} / \mathrm{H}]$ depletion which Pettini et al. (2002) has argued is further evidence for a system that has recently formed from the IGM. Erni et al. (2006) argue that the abundances are in good agreement with $10-50 M_{\odot}$ zero metallicity Pop III models.

Secondly, Cooke et al. (2010) identified a DLA towards the QSO J0035-0918 $(z=2.340)$ with a remarkably strong $[\mathrm{C} / \mathrm{Fe}]$ enhancement and with the same odd-even effect in light elements. This result is particularly striking because it is reminiscent of the recent discovery of carbon-enhanced extremely metal poor stars in the Galactic halo (Beers \& Christlieb 2005; Lucatello et al. 2006). They infer the total mass of neutral gas to be less than $3 \times 10^{6} M_{\odot}$ within a linear scale of less than $100 \mathrm{pc}$. The inferred amount of carbon from the $n_{\mathrm{H}}$ density limit is consistent with one or two SN enrichment events. These rough physical parameters are in line with our M 70 model presented in Figure 1(vi). Here we see that most sight 
lines through the core of our model $\left(r<r_{s}\right)$ would have been selected as a DLA system with $[\mathrm{Fe} / \mathrm{H}] \approx-3$.

JBH is supported by a Federation Fellowship from the Australian Research Council (ARC), which also funds TK's research position. RSS's research is supported by ARC DP grant DP0664434, Feedback Processes in Galaxy Formation. JBH is indebted to Merton College, Oxford for a Visiting Research Fellowship, and to the Leverhulme Trust for a Visiting Professorship to Oxford. JBH and TK are grateful to the BIPAC Institute for their hospitality.

\section{References}

Abel, T., et al., 2002, Science, 295, 93

Barkana, R., \& Loeb, A., 1999, ApJ, 523, 54

Beers, T., \& Christlieb, N., 2005, ARA\&A, 43, 531

Bland-Hawthorn, J., et al., 2011, ApJ, in press

Bland-Hawthorn, J., et al., 2007, ApJ, 670, L109

Clark, P., et al., 2008, ApJ, 672, 757

Cooke, R., et al., 2010, MNRAS, in press [arXiv:1011.0733]

Efstathiou, G., 1992, MNRAS, 256, 43P

Erni, P., et al., 2006, A\&A, 451, 19

Gnedin, N., 2000, ApJ, 542, 535

Heger, A., \& Woosley, S., 2002, ApJ, 567, 532

Karlsson, T., et al., 2011, Rev. Mod. Phys., in press

Kirby, E., et al., 2010, ApJS, 191, 352

Lucatello, S., et al., 2006, ApJ, 652, L37

McGaugh, S., et al., 2010, ApJ, 708, L14

MacLow, M., \& Ferrara, A., 1999, 513, 142

Martin, N., et al., 2008, ApJ, 684, 1075

Nichols, M., \& Bland-Hawthorn, J., 2009, ApJ, 707, 1642

Okamoto, T., et al., 2008, MNRAS, 390, 920

Okrochkov, M., \& Tumlinson, J., 2010, ApJ, 716, L41

Pancino, E., et al., 2010, A\&A, in press [arXiv:1012.4756]

Peebles, P., \& Dicke, R., 1968, ApJ, 154, 891

Peebles, P., 1993, Principles of Physical Cosmology (PUP)

Pettini, M., et al., 2002, A\&A, 391, 21

Pettini, M., et al., 2008, MNRAS, 391, 1499

Penprase, B., et al., 2010, ApJ, 721, 1P

Strigari, L., et al., 2008, ApJ, 678, 614

Sutherland, R., 2010, Ap\&SS, 327, 173

Tolstoy, E., et al., 2009, ARAA, 47, 371

Tsuribe, T., \& Omukai, K., 2008, ApJ, 676, L45

Walker, M., et al., 2009, ApJ, 704, 1274 